\title{
Resilience in the Air Traffic Management Arena
}

\author{
Gary Lohr \\ NASA Langley
}




\section{Objectives}

- Provide a perspective of how "resilience" relates to air traffic management

- Provide a perspective of the role of research in system recovery 


\section{Designing an Air Transportation System with Multi-Level Resilience Antlers Hilton, Colorado Springs, CO - October 5-9, 2014

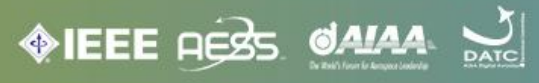

\section{Conference and Plenary Theme - "Resilience"}

Resilience is...

"Graceful and expected degradation with planned and achievable recovery such that no one component drives the "health" of the overall system unless required" 


\section{Designing an Air Transportation System with Multi-Level Resilience \\ Antlers Hilton, Colorado Springs, CO - October 5-9, 2014

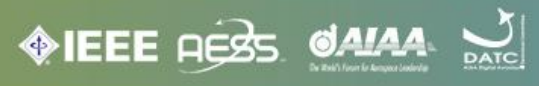

A degradation of capabilities within the NAS occurs on a daily basis at varying levels of severity.

- As an "expected" event, black and white often times morphs to shades of gray

- Notion of "components" casts a wide net 


\section{Human Factors Viewpoint of Resilient Systems}

- Systems that-

- Know what to do - addresses the actual

- Know what to look for - addresses the critical

- Know what to expect - addresses the potential

- Know what has happened - addresses the factual, learning from past

- What they look like-

- Appropriate information provided to allow humans SA of above

- Clearly defined and communicated roles and authority levels (including back-ups)

- Supported communication among agents

- Flexible function allocation among human and automated agents 


\section{Resilience as Matter of Perspective}

- User (airlines, general aviation, military)

- Air Traffic Service Provider (controller, traffic manager, ...)

- Airport operators

- Pilots

- Passengers 


\section{Resilience as Matter of Scale}

- Airport surface

- Local (terminal) Airspace

- Airspace (enroute) - national level 


\section{Factors Affecting Resiliency in ATM}

- Traffic Flow Management

- Constraints

- Competing interests

- Changing landscape (e.g., FAR 117)

- System capabilities 


\section{Designing an Air Transportation System with Multi-Level Resilience \\ Antlers Hilton, Colorado Springs, CO - October 5-9, 2014 4. IEEE RESS. OAIAA WOAC}

\section{Bridging the Gap...}

- Convergence of differing objectives

- More effective avenues for exchange of these objectives

- More effective tools (?automation/processing?) for processing options

- Longer planning horizons

- Research to address the recovery process 


\section{Designing an Air Transportation System with Multi-Level Resilience \\ Antlers Hilton, Colorado Springs, CO - October 5-9, 2014

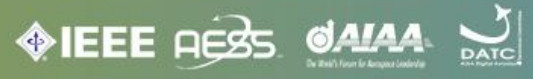

\section{Relationship Between Research and Resilience}

Research plays a vital role in improving resilience in air traffic management although tools/concepts are not normally investigated with a resilience focus. However, research is the key to development/refinement of requisite NAS capabilities that will serve, by their inherent objectives, to improve resiliency in the System. 


\section{Designing an Air Transportation System with Multi-Level Resilience \\ Antlers Hilton, Colorado Springs, CO - October 5-9, 2014

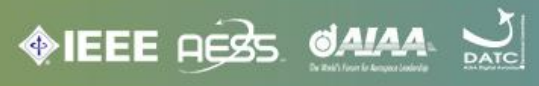

\section{Examples of Research Affecting System Resilience}

- Wake vortex

- Dynamic Airspace Configuration

- Precision Release of Departures

- Traffic Flow Management Enhancements 


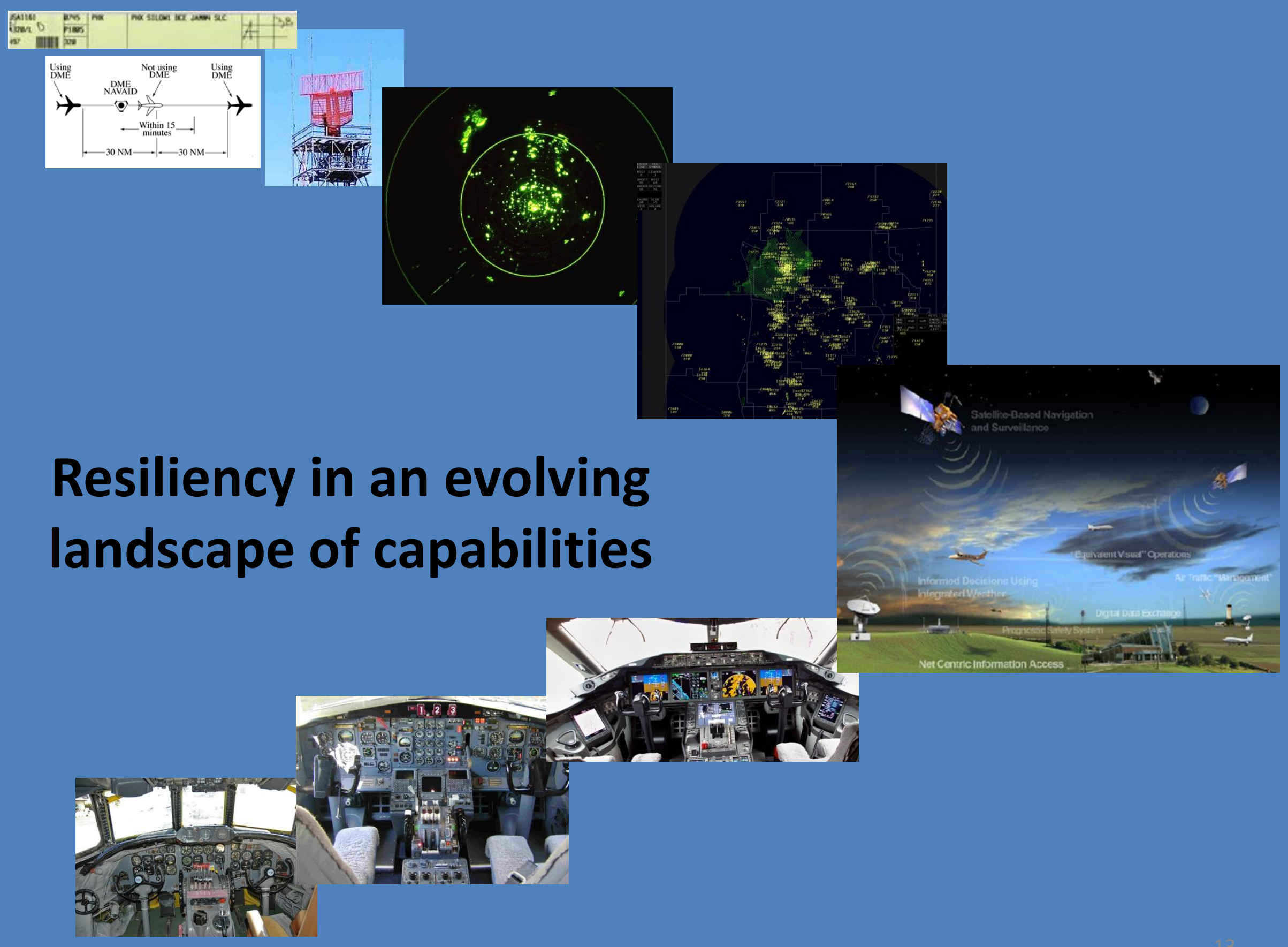




\section{Summary}

- Resilience defined

- Nature of resilient systems

- The NAS: "players", scope/environment

- Key factors affecting system resilience

- Role of research in enhancing resiliency 
